\title{
Relationship between serum uric acid level and mild cognitive impairment in Chinese community elderly
}

Miao Liu ${ }^{1,2^{*}+}$, Jianhua Wang ${ }^{1,2+}$, Jing Zeng ${ }^{1,2}$ and Yao He $\mathrm{H}^{1,2,3}$

\begin{abstract}
Background: To evaluate the association between SUA levels within a normal to high range and the risk of mild cognitive impairment (MCl) among community elderly.

Methods: The present study was based on 2102 community elderly from a cross-sectional study conducted in a representative urban area of Beijing between 2009 and 2010. The mean age were $71.2 \pm 6.6$ years old, 59.7\% were female. Mini-Mental State Examination (MMSE) was used to assess cognitive function by trained neurology doctors.

Results: The prevalence of hyperuricemia and $\mathrm{MCI}$ was $16.7 \%$ and $15.9 \%$ respectively. With the increase of SUA levels, the prevalence of $\mathrm{MCl}$ showed a strong decreasing linear trend. Multiple logistic regression analysis showed ORs for MCl were 1.01(95\% Cl: 0.69-1.48), 1.50(95\% Cl: 0.85-2.64), 1.65(95\% Cl: 1.12-2.43) and 1.53(95\% Cl: 1.00-2.33), 1.84(95\% Cl: 1.27-2.90), 1.92(95\% Cl: 1.02-3.35) for the second, third and highest quarters among men and women respectively (with the lowest quartile as the reference).
\end{abstract}

Conclusions: Higher SUA levels, when in the normal range, were positively associated with cognitive function among Chinese community elderly, but this association was not robust among participants with hyperuricemia.

Keywords: Chinese, Elderly, Epidemiology, Mild cognitive impairment, Uric acid

\section{Background}

With the acceleration in the population aging, dementia has become one of the most important health problems over the world [1]. Studies have estimated that the total number of population affected by dementia is about 46.8 million globally, and the number keeps increasing $[2,3]$. According to WHO' report, the patients affected by dementia will double every 20 years, and most of them live in developing countries [4]. In China, dementia is also a serious problem along with the large elder population. It was estimated people with dementia in China increased from 3.68 million in 1990 to 9.19 million in 2010 [5]. On the other hand, the disease burden of dementia is huge. It will not only cause disability, reduce life quality of patients, and also causes burdens to caregivers and take

\footnotetext{
* Correspondence: liumiaolmbxb@163.com

${ }^{\dagger}$ Equal contributors

'Institute of Geriatrics, Chinese PLA General Hospital, 28 Fuxing Road, Beijing 100853, China

${ }^{2}$ Beijing Key Laboratory of Aging and Geriatrics, Beijing, China

Full list of author information is available at the end of the article
}

up health and social care resources [6-8]. Hence, it's of critical importance to carry out early prevention and control of dementia.

Several studies have focused on serum uric acid (SUA) level and cognitive function [9-15]. Like the old saying, gout unlike any other disease, kills more wise men than simple, which means that gout patients have higher SUA levels and also higher intelligence quotient (including cognitive function) [16].Although a number of studies have revealed the relationship, the conclusion remains controversial. Some studies found that higher SUA level was beneficial to cognitive function and prevent cognitive impairment $[12,13]$. But other studies also came to the opposite conclusion; SUA was a risk factor for cognitive function, like for cardiovascular disease [10]. Also, most studies only included the normal SUA levels, but missed the very high SUA level [17-19]. Thus, SUA levels within a normal range have not been fully studied with cognitive function. Therefore, we examined the association of SUA level in the normal range and also in 
the hyperuricemia range for related risk of $\mathrm{MCI}$ in an urban community elderly population of Beijing, China.

\section{Methods \\ Study population}

The sampling methods were reported in our previous study [20]. In brief, this was a population-based crosssectional survey conducted in a metropolitan area representative of urban community in Beijing, China. A two-stage stratified clustering sampling method was used. First, 9 residents were randomly selected from the total 94 residents in Wanshoulu district. Second, all the individuals aged $\geq 60$ years that had lived in the selected residence for more than 1 year were recruited. A total of 2162 residents aged 60-95 years were selected and invited for screening. Besides the 60 elderly with incomplete data, the left 2102 residents (848 men, 1254 women) completed the survey, and the participants accounted for about $10 \%$ of total elderly in Wanshoulu community.

All the nurses and doctors who took part in the field survey had strict training. Face-face interview was carried out, all the detail health condition of the participants were asked using a standardized questionnaire, including demographic factors, medical history, family history, and lifestyles. Physical examinations was carried out by specially trained nurses, including height, weight, waist circumstance (WC). We also calculated body mass index (BMI). Two blood pressures were measured using sphygmomanometer in a sitting position after 30 min' rest. Fasting blood specimens of all participants were obtained for standard tests of glucose, blood lipids and SUA level.

\section{Definitions}

Hyperuricemia was defined positive if SUA level was $\geq 7.0 \mathrm{mg} / \mathrm{dL}(417 \mu \mathrm{mol} / \mathrm{L})$ in men or $\geq 6.0 \mathrm{mg} / \mathrm{dL}$ $(357 \mu \mathrm{mol} / \mathrm{L})$ in women, or positively diagnosed [21]. SUA levels of participants with and without hyperuricemia were categorized into 4 levels using the quartiles $\left(\mathrm{P}_{25}, \mathrm{P}_{50}\right.$ and $\left.\mathrm{P}_{75}\right)$ as cut-off values by gender.

We used the Mini-Mental State Examination (MMSE) to assess cognitive function by trained neurology doctors. The MMSE scale was proved to be feasible among Chinese for dementia screening [22]. MCI was defined according the guideline, which is as follows: (1) for illiteracy participants, MMSE score less than a score of 17; (2) for participants with 1-6 education years, MMSE score less than a score of 20; (3) for participants with more than 7 education years, MMSE score less than a score of 24 [23, 24].

Prevalence of cerebral vascular disease and kidney disease was defined according to previous medical diagnosis by hospitals of second level and above. Current smoking was defined as those who currently smoked at least one cigarette daily; current alcohol drinking was defined as drinking alcohol at least once weekly for the past year. Physical activity was divided into two categories: $\geq 0.5 \mathrm{~h} /$ day or $<0.5 \mathrm{~h} /$ day.

\section{Statistical analysis}

For the basic characteristics, $\overline{\mathrm{x}} \pm \mathrm{s}$ were used for continuous variables like age, WC, BMI, blood pressure, blood lipid, glycaemia and SUA level. $n$ (\%) were used for categorical variables like gender(male or female), education level (less than 6 years, or more than 6 years), marriage status (married or unmarried), current smoking (yes or no), current drinking(yes or no), physical activity $\geq 0.5 \mathrm{~h} /$ day (yes or no), family history (yes or no). $\mathrm{T}$ test and Chisquare test were used to examine differences in continuous and categorical variables. Linear multiple regression and logistic multivariable regression was used to estimate the $\beta$ and odds ratio (OR) of gender-specific SUA level with cognitive function. All analyses were conducted using SPSS (19.0, No. of Serial: 5,076,595).

\section{Results \\ Clinical features of participants with and without hyperuricemia}

The mean SUA level was $5.3 \pm 1.4 \mathrm{mg} / \mathrm{dL}$ (range, $1.4-11.3 \mathrm{mg} / \mathrm{dL}$ ), men had a higher SUA level than women $(5.8 \pm 1.3$ vs $5.2 \pm 1.4, p<0.001)$. The general characteristics according to gender and hyperuricemia were shown in Table 1. The mean age were $71.2 \pm 6.6$ years old, $59.7 \%$ were female. The education level was high, $72.4 \%$ of all the participants had at least gone to junior middle school. Participants with hyperuricemia had older age, greater WC, higher BMI, higher TG, level and lower HDL-C levels. However, there were no significant differences in blood pressure and FPG. In women, besides these differences, we also observed higher levels of 2hPG in participants with hyperuricemia than those without. The percentage of participants who were married, better educated, current smoking and drinking did not differ with hyperuricemia. For MMSE score, participants with hyperuricemia had higher MMSE score in men $(27.7 \pm 2.6$ vs $27.0 \pm 3.8, p=0.014)$ but not in women $(27.0 \pm 3.5$ vs $26.7 \pm 3.8, p=0.057)$.

\section{Age and gender-specific prevalence of hyperuricemia and $\mathrm{MCl}$ of the study participants}

The prevalence of hyperuricemia and $\mathrm{MCI}$ in this community elderly was $16.7 \%$ and $15.9 \%$ respectively. Prevalence of both two diseases increased linearly with increasing age group $(p<0.001)$. Women have a statistically higher prevalence of $\mathrm{MCI}$ than men $(18.0 \%$ vs. $12.9 \%, P=0.001)$. For hyperuricemia, men had a relatively higher prevalence in the elderly aged less than 70 yrs., but women had increased at a faster rate than men, however, the difference was not statistically significance $(p>0.05)$. The results were listed in Table 2. 
Table 1 General characteristics of the participants by hyperuricemia

\begin{tabular}{|c|c|c|c|c|c|c|c|c|c|}
\hline \multirow[t]{2}{*}{ Characteristic } & \multicolumn{4}{|l|}{ Male $(n=848)$} & \multicolumn{4}{|c|}{ Female $(n=1254)$} & \multirow{2}{*}{$\begin{array}{l}\text { Total } \\
(n=2102)\end{array}$} \\
\hline & $\begin{array}{l}\text { hyperuricemia } \\
(n=148)\end{array}$ & $\begin{array}{l}\text { No hyperuricemia } \\
(n=700)\end{array}$ & $P$ & subtotal & $\begin{array}{l}\text { hyperuricemia } \\
(n=204)\end{array}$ & $\begin{array}{l}\text { No hyperuricemia } \\
(n=1050)\end{array}$ & $P$ & subtotal & \\
\hline \multicolumn{10}{|l|}{$\bar{x} \pm s$} \\
\hline Age (yrs) & $73.5 \pm 6.9$ & $72.1 \pm 6.8$ & 0.028 & $72.3 \pm 6.9$ & $71.8 \pm 6.0$ & $70.1 \pm 6.3$ & 0.001 & $70.4 \pm 6.2$ & $71.2 \pm 6.6$ \\
\hline$W C(\mathrm{~cm})$ & $94.0 \pm 8.4$ & $90.6 \pm 8.4$ & $<0.001$ & $91.2 \pm 8.5$ & $90.3 \pm 9.5$ & $85.6 \pm 8.6$ & $<0.001$ & $86.4 \pm 8.9$ & $88.3 \pm 9.1$ \\
\hline BMI(kg/m2) & $25.7 \pm 3.0$ & $24.8 \pm 3.1$ & 0.002 & $24.9 \pm 3.1$ & $26.4 \pm 3.8$ & $24.8 \pm 3.5$ & $<0.001$ & $25.0 \pm 3.6$ & $25.0 \pm 3.4$ \\
\hline $\mathrm{SBP}(\mathrm{mm} \mathrm{Hg})$ & $136.8 \pm 17.5$ & $136.6 \pm 18.0$ & 0.927 & $136.7 \pm 17.9$ & $141.9 \pm 20.4$ & $140.2 \pm 20.9$ & 0.285 & $140.5 \pm 20.8$ & $139.0 \pm 19.8$ \\
\hline $\mathrm{DBP}(\mathrm{mmHg})$ & $77.4 \pm 9.4$ & $78.6 \pm 9.7$ & 0.191 & $78.4 \pm 9.7$ & $75.9 \pm 10.4$ & $76.8 \pm 9.9$ & 0.273 & $76.6 \pm 10.0$ & $77.3 \pm 9.9$ \\
\hline $\mathrm{TC}(\mathrm{mmol} / \mathrm{L})$ & $5.0 \pm 1.1$ & $4.9 \pm 0.9$ & 0.131 & $4.9 \pm 0.9$ & $5.5 \pm 1.0$ & $5.5 \pm 1.0$ & 0.550 & $5.5 \pm 1.0$ & $5.3 \pm 1.0$ \\
\hline $\mathrm{TG}(\mathrm{mmol} / \mathrm{L})$ & $1.8 \pm 1.0$ & $1.5 \pm 0.8$ & 0.001 & $1.5 \pm 0.9$ & $2.1 \pm 1.0$ & $1.7 \pm 0.9$ & $<0.001$ & $1.8 \pm 0.9$ & $1.7 \pm 0.9$ \\
\hline $\mathrm{HDL}-\mathrm{C}(\mathrm{mmol} / \mathrm{L})$ & $1.2 \pm 0.3$ & $1.3 \pm 0.4$ & 0.016 & $1.3 \pm 0.4$ & $1.4 \pm 0.4$ & $1.5 \pm 0.4$ & $<0.001$ & $1.5 \pm 0.4$ & $1.4 \pm 0.4$ \\
\hline $\mathrm{LDL}-\mathrm{C}(\mathrm{mmol} / \mathrm{L})$ & $3.1 \pm 0.9$ & $3.0 \pm 0.8$ & 0.338 & $3.1 \pm 0.8$ & $3.4 \pm 0.8$ & $3.4 \pm 0.9$ & 0.506 & $3.4 \pm 0.9$ & $3.3 \pm 0.8$ \\
\hline $\mathrm{FPG}(\mathrm{mmol} / \mathrm{L})$ & $6.0 \pm 1.3$ & $6.1 \pm 1.6$ & 0.428 & $6.1 \pm 1.5$ & $6.1 \pm 1.3$ & $6.1 \pm 1.8$ & 0.704 & $6.1 \pm 1.8$ & $6.1 \pm 1.7$ \\
\hline $2 \mathrm{hPG}(\mathrm{mmol} / \mathrm{L})$ & $8.1 \pm 2.6$ & $8.2 \pm 3.0$ & 0.707 & $8.2 \pm 3.0$ & $9.2 \pm 3.3$ & $8.2 \pm 3.3$ & $<0.001$ & $8.4 \pm 3.3$ & $8.3 \pm 3.2$ \\
\hline MMSE score & $27.7 \pm 2.6$ & $27.0 \pm 3.8$ & 0.014 & $27.1 \pm 3.6$ & $27.0 \pm 3.5$ & $26.7 \pm 3.8$ & 0.057 & $26.8 \pm 3.7$ & $26.9 \pm 3.7$ \\
\hline \multicolumn{10}{|l|}{$n(\%)$} \\
\hline \multicolumn{10}{|l|}{ Education $\geq 7 \mathrm{yrs}$} \\
\hline Married & $131(88.5)$ & $650(92.9)$ & 0.075 & $781(92.1)$ & 157(77.0) & 836(79.6) & 0.392 & $993(79.2)$ & $1774(84.4)$ \\
\hline Current smoking & $27(18.2)$ & 153(21.9) & 0.329 & $180(21.2)$ & $7(3.4)$ & $44(4.2)$ & 0.615 & $51(4.1)$ & 231(11.1) \\
\hline $\begin{array}{l}\text { Current alcohol } \\
\text { drinking }\end{array}$ & $58(39.2)$ & 267(38.1) & 0.812 & $325(38.3)$ & $14(6.9)$ & $86(8.2)$ & 0.522 & $100(8.0)$ & $425(20.2)$ \\
\hline $\begin{array}{l}\text { Physical activity } \\
(\geq 0.5 \mathrm{~h} / \text { day })\end{array}$ & $88(59.5)$ & $422(60.3)$ & 0.852 & $510(60.1)$ & $121(59.3)$ & $603(57.4)$ & 0.618 & $724(57.7)$ & $1234(58.7)$ \\
\hline $\begin{array}{l}\text { Family history } \\
\text { of dementia }\end{array}$ & $10(6.8)$ & $32(4.6)$ & 0.266 & $42(5.1)$ & $10(4.9)$ & $50(4.8)$ & 0.932 & $60(4.8)$ & $102(4.9)$ \\
\hline $\begin{array}{l}\text { Cerebral vascular } \\
\text { disease }\end{array}$ & 28(18.9) & $90(12.9)$ & 0.053 & 118(13.9) & $25(12.3)$ & 124(11.8) & 0.857 & 149(11.9) & $267(12.7)$ \\
\hline Kidney disease & $31(20.9)$ & $52(7.4)$ & $<0.001$ & $83(9.8)$ & $5(2.5)$ & $17(1.6)$ & 0.408 & $22(1.8)$ & $105(5.0)$ \\
\hline
\end{tabular}

Data are mean \pm SD for continuous values or $\mathrm{n}(\%)$ for category values

$T C$ total cholesterol, $T G$ triglyceride, $L D L-C$ low density lipoprotein cholesterol

Mean MMSE score and prevalence of $\mathrm{MCl}$ for participants with and without hyperuricemia

The mean MMSE score showed an increasing trend along with quarters of SUA levels among those without hyperuricemia in both genders ( $\mathrm{p}$ for trend $<0.05$ ). The prevalence of MCI also showed the same trend, which decreased from $20.9 \%$ to $13.7 \%(p=0.030)$. Among those participants who had hyperuricemia, the mean score of MMSE and the prevalence of MCI did not show statistically significant changing trend with SUA levels. The results were listed in Table 3. Also, we can see from Table 3, the mean score kept increasing for the first two quarters among

Table 2 Prevalence of hyperuricemia and $\mathrm{MCl}$ by gender and age group

\begin{tabular}{|c|c|c|c|c|c|c|c|c|}
\hline & \multicolumn{4}{|c|}{ Prevalence of hyperuricemia (\%) } & \multicolumn{4}{|c|}{ Prevalence of $\mathrm{MCl}(\%)$} \\
\hline & Men & Female & $p$ & Subtotal & Men & Female & $p$ & Subtotal \\
\hline \multicolumn{9}{|l|}{ Age group } \\
\hline $60-69$ yrs & 14.8 & 12.3 & 0.300 & 13.2 & 7.4 & 9.9 & 0.048 & 9.0 \\
\hline $70-79$ yrs & 17.8 & 18.7 & 0.704 & 18.3 & 12.9 & 22.4 & $<0.001$ & 18.5 \\
\hline$\geq 80 \mathrm{yrs}$ & 22.6 & 24.1 & 0.815 & 23.2 & 26.1 & 38.0 & 0.039 & 30.9 \\
\hline Ptrend & 0.036 & 0.002 & & $<0.001$ & $<0.001$ & $<0.001$ & & $<0.001$ \\
\hline subtotal & 17.5 & 16.3 & 0.475 & 16.7 & 12.9 & 18.0 & 0.001 & 15.9 \\
\hline
\end{tabular}


Table 3 MMSE score and MCI Prevalence According to SUA quartiles by gender and hyperuricemia

\begin{tabular}{|c|c|c|c|c|c|c|}
\hline & \multicolumn{6}{|c|}{ SUA quartiles by gender and hyperuricemia } \\
\hline & $\overline{Q_{1}}$ & $\mathrm{Q}_{2}$ & $\mathrm{Q}_{3}$ & $\mathrm{Q}_{4}$ & $P_{\text {trend }}$ & total \\
\hline \multicolumn{7}{|c|}{ Participants without hyperuricemia } \\
\hline \multicolumn{7}{|l|}{ MMSE score } \\
\hline Male & $26.9 \pm 3.9$ & $27.0 \pm 4.0$ & $27.1 \pm 3.4$ & $27.2 \pm 3.7$ & 0.038 & $27.0 \pm 3.8$ \\
\hline Female & $26.3 \pm 3.8$ & $26.5 \pm 4.1$ & $27.0 \pm 3.9$ & $27.1 \pm 3.2$ & 0.048 & $26.7 \pm 3.8$ \\
\hline Subtotal & $26.6 \pm 3.9$ & $26.8 \pm 4.1$ & $27.0 \pm 3.7$ & $27.1 \pm 3.4$ & 0.031 & $26.8 \pm 3.8$ \\
\hline \multicolumn{7}{|c|}{ Prevalence of $\mathrm{MCl}$} \\
\hline Male & $28(15.7)$ & $23(13.7)$ & 24(13.6) & $21(11.9)$ & 0.039 & $96(13.7)$ \\
\hline Female & $68(25.8)$ & $52(20.1)$ & $39(14.8)$ & $36(13.6)$ & 0.008 & 195(18.6) \\
\hline Subtotal & $96(20.9)$ & $75(16.7)$ & $63(15.2)$ & $57(13.7)$ & 0.030 & $291(16.6)$ \\
\hline \multicolumn{7}{|c|}{ Participants with hyperuricemia } \\
\hline \multicolumn{7}{|l|}{ MMSE score } \\
\hline Male & $27.8 \pm 3.2$ & $27.7 \pm 2.5$ & $27.7 \pm 2.8$ & $27.5 \pm 2.2$ & 0.965 & $27.7 \pm 2.6$ \\
\hline Female & $26.7 \pm 3.6$ & $27.4 \pm 2.7$ & $27.2 \pm 4.1$ & $26.5 \pm 3.4$ & 0.549 & $27.0 \pm 3.5$ \\
\hline Subtotal & $27.2 \pm 3.4$ & $27.5 \pm 2.5$ & $27.3 \pm 3.6$ & $27.0 \pm 3.1$ & 0.772 & $27.2 \pm 3.1$ \\
\hline \multicolumn{7}{|c|}{ Prevalence of $\mathrm{MCl}$} \\
\hline Male & $5(13.5)$ & $3(8.1)$ & $4(10.8)$ & $1(2.7)$ & 0.155 & $13(8.8)$ \\
\hline Female & $9(18.8)$ & $7(13.0)$ & $4(7.8)$ & $11(21.6)$ & 0.854 & $31(15.2)$ \\
\hline Subtotal & $14(16.5)$ & $10(11.0)$ & $8(9.1)$ & $12(13.6)$ & 0.526 & $44(12.5)$ \\
\hline
\end{tabular}

those with hyperuricemia, and got decreased for the highest two quarters of SUA levels. The prevalence also decreased from $16.5 \%$ to $9.1 \%$ for the first three quarters, and changed to $13.6 \%$ for the highest quarter of SUA level.

\section{Multivariate associations of SUA level with MMSE score and $\mathrm{MCl}$ prevalence}

Table 4 showed the multivariate associations between SUA and with MMSE score and MCI prevalence. This table showed that after adjusted age, gender, education, marital status, current smoking, current alcohol drinking, BMI, physical activity $\geq 0.5 \mathrm{~h} /$ day, family history of dementia, hypertension, cerebral vascular disease and diabetes, participants with higher SUA levels were at significantly elevated risk for MCI prevalence among those without hyperuricemia. For MMSE score, the $\beta$ (95\% CI) of SUA were $0.14(95 \%$ CI: $0.04-0.42)$ and 0.28 (95\% CI: $0.02-0.53$ ) for men and women respectively. For MCI prevalence, the OR $(95 \% \mathrm{CI})$ of quarters of SUA showed an increasing trend. The ORs were

Table 4 Multiple linear and logistic regression of SUA level for MMSE score and MCI prevalence

\begin{tabular}{|c|c|c|c|c|c|c|c|}
\hline \multirow[b]{2}{*}{ MMSE score } & \multicolumn{2}{|c|}{ SUA level (continuous) } & \multicolumn{4}{|c|}{ SUA level (quartiles by gender) } & \multirow[b]{2}{*}{$P_{\text {trend }}$} \\
\hline & $\beta(95 \% C l)$ & $P$ & Q1 & Q2 $\beta(95 \% \mathrm{Cl})$ & Q3 $\beta(95 \% \mathrm{Cl})$ & Q4 $\beta(95 \% \mathrm{Cl})$ & \\
\hline \multicolumn{8}{|c|}{ Participants with hyperuricemia } \\
\hline Male & $-0.03(-0.56-0.39)$ & 0.721 & 1.00 (Ref) & $0.97(0.66-1.43)$ & $0.92(0.58-1.26)$ & $0.99(0.87-1.13)$ & 0.544 \\
\hline Female & $-0.01(-0.43-0.37)$ & 0.882 & 1.00(Ref) & $1.07(0.80-1.27)$ & $1.05(0.78-1.31)$ & $1.00(0.92-1.18)$ & 0.922 \\
\hline \multicolumn{8}{|c|}{ Participants without hyperuricemia } \\
\hline Male & $0.14(0.04-0.42)$ & 0.048 & 1.00 (Ref) & $1.01(0.69-1.48)$ & $1.50(0.85-2.64)$ & $1.65(1.12-2.43)$ & 0.027 \\
\hline Female & $0.28(0.02-0.53)$ & 0.037 & 1.00 (Ref) & $1.53(1.00-2.33)$ & $1.84(1.27-2.90)$ & $1.92(1.02-3.35)$ & 0.017 \\
\hline Prevalence of $\mathrm{MCl}$ & OR (95\% Cl) & $P$ & Q1 & Q2 OR (95\% Cl) & Q3 OR (95\% Cl) & Q4 OR (95\% Cl) & $P_{\text {trend }}$ \\
\hline \multicolumn{8}{|c|}{ Participants with hyperuricemia } \\
\hline Male & $1.06(0.47-1.64)$ & 0.126 & 1.00(Ref) & $0.48(0.10-2.33)$ & $0.54(0.12-2.40)$ & $0.10(0.01-0.97)$ & 0.256 \\
\hline Female & $1.02(0.67-1.56)$ & 0.915 & 1.00 (Ref) & $0.59(0.18-1.90)$ & $0.32(0.08-1.23)$ & $1.08(0.36-3.20)$ & 0.246 \\
\hline \multicolumn{8}{|c|}{ Participants without hyperuricemia } \\
\hline Male & $0.82(0.68-0.99)$ & 0.047 & 1.00(Ref) & $0.95(0.50-1.78)$ & $0.91(0.49-1.70)$ & $0.72(0.38-1.36)$ & 0.025 \\
\hline Female & $0.80(0.65-0.97)$ & 0.034 & 1.00 (Ref) & $0.87(0.45-1.70)$ & $0.72(0.44-1.77)$ & $0.64(0.39-1.06)$ & 0.008 \\
\hline
\end{tabular}

Adjusted for age, education, marital status, BMI, current smoking, current drinking, physical activity $\geq 0.5$ h/day, family history of dementia, hypertension, cerebral vascular disease and diabetes 
1.01(95\% CI: $0.69-1.48), \quad 1.50(95 \% \quad$ CI: $0.85-2.64)$, 1.65(95\% CI: $1.12-2.43)$ and $1.53(95 \% \mathrm{CI}: 1.00-2.33)$, 1.84(95\% CI: $1.27-2.90)$, $1.92(95 \%$ CI: $1.02-3.35)$ for the second, third and highest quarters among men and women respectively (with the lowest quartile as the reference). Among the participants with hyperuricemia, the $\beta$ (95\% CI) for MMSE score were -0.03(95\% CI: $-0.56-0.39)$ and $-0.01(95 \%$ CI: $-0.43-0.37)$ for men and women. In the model of MCI prevalence, the ORs were also not statistically significant among participants with hyperuricemia.

We also ascertained the association of SUA level with MMSE score and MCI prevalence in the sensitivity analysis and the results were similar (Additional file 1: Appendix Table S1 and S2). When participants with cerebral vascular disease $(n=267,12.7 \%)$ and kidney disease $(n=105,5.0 \%)$ were excluded, the $\beta$ s and ORs were similar with that in the Table 4. Higher SUA level was related with better cognitive function when SUA was in the normal range. There was also still no significant association among participants with hyperuricemia.

\section{Discussion}

In this representative community elderly based study; we evaluated the association of SUA level and cognitive function. The results that higher SUA level within normal range were positively associated with a decreased prevalence of MCI even after adjusting for demographic and other potential confounders. However, this association was not significant among participants with hyperuricemia.

Different from the high risk and harm of SUA level and cardiovascular diseases [25], a number of studies have provided evidence that SUA had protective effect on cognitive function and is beneficial to reduce dementia [14-16]. In this representative sample of Chinese urban elderly, we also found a graded positive association between hyperuricemia and cognitive function. When in the normal range, higher SUA level was associated with higher MMSE score and lower risk of MCI prevalence. And cognitive function was found to rise along with the increase of quarters of SUA levels. This is in accordance with previous studies. Molshatzki found that after 10 years follow-up, low uric acid levels in patients with preexisting cardiovascular disease are associated with poorer cognitive function [14]. MéndezHernández revealed that higher levels of uric acid are associated with a decreased risk of dementia using a case-control study in a Mexican population [13]. Yili Wu found that among Chinese aged 50-74 years, prevalence of cognitive disorder declined across UA tertiles [9]. The mechanism between higher SUA level and better cognitive function was not clear; one possible explanation was oxidative stress. SUA is a water-soluble antioxidant, which accounts for more than half of the free radical scavenging activity [17]. SUA could exert neuro-protective effects against Alzheimer's disease via its antioxidant capacities [16].
The present study also provides evidence that elevated SUA level was not associated with MCI among those participants with hyperuricemia. When SUA level was higher than the normal range, there was no statistically significant positive correlation with cognitive function. This result confirmed previous studies. A study about elderly women from U.S. showed higher baseline SUA levels was associated with poorer cognitive performance $(p=0.048)$. Another study from Italy showed that participants who had the highest SUA tertile had higher risk $(\mathrm{OR}=3.32$, 95\%CI: $1.06-10.42)$ to suffer from dementia based on 1016 community-dwellings. On the other hand, there were several studies revealed that higher SUA levels were related to higher prevalence of gout and cardiovascular diseases $[26,27]$. So the key question is what is the appreciate cut point for SUA levels. There is urgent need for long-time follow-up cohort studies to verify the optimal diagnostic boundary value, which would maximize the benefits, not only for gout and cardiovascular diseases, but also for MCI and dementia.

The study had several potential limitations. First, the SUA level was measured once, and may not reflect the true value, and did not consider the fluctuation. Second, the limitations of cross-sectional study lowered strength of evidence for causal inference. Long time follow up study are needed to confirm the results. Third, results among hyperuricemia individuals are based on very few cases of MCI patients, thus results might not be reliable. Fourth, there were several possible confounding factors we didn't consider ApoE genotype and drug treatment status. On the other hand, the study also some advantages. We used strict training process and quality assurance programs during the whole study. The selected community was representative metropolitan area of the geographic and economic characteristics in Beijing, and the response rate was high. So this population-based study with large sample and high response rate would have a good chance to better analysis the association between SUA level and cognitive function. Second, the SUA level in this study was carefully analyzed using the normal range and higher SUA level in hyperuricemia, which is different from most previous studies. The results also showed that it's not the simple "the higher the better" or "no correlation" or "the lower the better". The association results were different with SUA levels.

\section{Conclusion}

In summary, the present study showed higher SUA level are related to lower prevalence of $\mathrm{MCI}$ and better cognitive function, only when SUA level was in the normal range among Chinese community elderly population. This indicated that further studies should focus on the appropriate SUA level, which is helpful to reduce both dementia and cardiovascular diseases. 


\section{Additional file}

Additional file 1: Appendix Tables (appendix Table S1 and S2). Multiple linear and logistic regression of SUA level for MMSE score and MCI prevalence for participants without cerebral vascular disease or without kidney diseases. We ascertained the association of SUA level with MMSE score and $\mathrm{MCl}$ prevalence in the sensitivity analysis (Appendix Table S1 and S2). When participants with cerebral vascular disease ( $n=267,12.7 \%$ ) and kidney disease ( $n=105,5.0 \%)$ were excluded, the $\beta$ s and ORs were similar with that in the Table 4 (all participants). (DOC $62 \mathrm{~kb}$ )

\section{Acknowledgements}

We thank Nurse Kang, Head Nurse Wang from PLA general hospital for research assistance and all the participants in flied works.

\section{Funding}

This study is supported by research grants from Beijing Natural Science Foundation (7174350), Beijing Municipal Science and Technology Commission (Z161100005016021), National key research and development program (2016YFC1303603) and State Key Development Program of Basic Research of China (973 programs, 2013CB530800). The funding body had no involvement in any stage of the study.

\section{Availability of data and materials}

The data are kept at Institute of Geriatrics, Chinese PLA General Hospital, China. The datasets used and/or analyzed during the current study available from Professor Yao He (yhe301@sina.com) on reasonable request.

\section{Authors' contributions}

$\mathrm{ML}$ and $\mathrm{YH}$ contributed to the design of the study. All authors were involved in the analysis and interpretation of the data. JHW, and JZ conducted the statistical analysis. ML and $\mathrm{YH}$ worked on the drafting of the manuscript. All authors read and approved the final manuscript.

\section{Ethics approval and consent to participate}

Study was approved by the Ethics Committees of Chinese PLA General Hospita (EC0411-2001). A written informed consent was obtained from participants who could understand and write their names. For those with severe cognitive impairment, we explained the informed consent both to the participants and also to their responsible caregivers and got the consent with the help of caregivers.

\section{Consent for publication}

Not applicable.

\section{Competing interests}

The author (s) declared no conflicts of interest with respect to the research authorship, funding, and/or publication of this article.

\section{Publisher's Note}

Springer Nature remains neutral with regard to jurisdictional claims in published maps and institutional affiliations.

\section{Author details}

${ }^{1}$ Institute of Geriatrics, Chinese PLA General Hospital, 28 Fuxing Road, Beijing 100853, China. ${ }^{2}$ Beijing Key Laboratory of Aging and Geriatrics, Beijing, China. ${ }^{3}$ State Key Laboratory of Kidney Disease, Chinese PLA General Hospital, 28 Fuxing Road, Beijing 100853, China.

Received: 14 June 2017 Accepted: 19 July 2017

Published online: 01 August 2017

\section{References}

1. Lambert MA, Bickel H, Prince M, Fratiglioni L, Von Strauss E, Frydecka D, et al. Estimating the burden of early onset dementia; systematic review of disease prevalence. Eur J Neurol. 2014;21:563-9.

2. Prince $M$, Bryce $R$, Albanese $E$, Wimo A, Ribeiro W, Ferri CP. The global prevalence of dementia: a systematic review and metaanalysis. Alzheimers Dement. 2013;9:63-75.

3. Wortmann M. Dementia: a global health priority - highlights from an ADI and World Health Organization report. Alzheimers Res Ther. 2012;4:40.
4. Alzheimer's Association. 2014 Alzheimer's disease facts and figures. Alzheimers Dement. 2014;10(2):e47-92.

5. Chan KY, Wang W, Wu JJ, Liu L, Theodoratou E, Car J, et al. Epidemiology of Alzheimer's disease and other forms of dementia in China, 1990-2010: a systematic review and analysis. Lancet. 2013;381:2016-23.

6. Yu R, Chau PH, McGhee SM, Cheung WL, Chan KC, Cheung SH, et al. Trends in prevalence and mortality of dementia in elderly Hong Kong population: projections, disease burden, and implications for long-term care. Int J Alzheimers Dis. 2012:2012:406852.

7. Prince $M$, Acosta D, Ferri CP, Guerra M, Huang $Y$, Jacob KS, et al. The association between common physical impairments and dementia in low and middle income countries, and, among people with dementia, their association with cognitive function and disability. A 10/66 dementia research group population-based study. Int J Geriatr Psychiatry. 2011;26:511-9.

8. Li N, Zhang L, Du W, Pang L, Guo C, Chen G, et al. Prevalence of dementiaassociated disability among Chinese older adults: results from a national sample survey. Am J Geriatr Psychiatry. 2015;23:320-5.

9. Wu Y, Zhang D, Pang Z, Jiang W, Wang S, Tan Q. Association of serum uric acid level with muscle strength and cognitive function among Chinese aged 50-74 years. Geriatr Gerontol Int. 2013;13:672-7.

10. Vannorsdall TD, Kueider AM, Carlson MC, Schretlen DJ. Higher baseline serum uric acid is associated with poorer cognition but not rates of cognitive decline in women. Exp Gerontol. 2014;60:136-9.

11. Gonzalez-Aramburu I, Sanchez-Juan P, Sierra M, Fernandez-Juan E, SanchezQuintana C, Berciano J, et al. Serum uric acid and risk of dementia in Parkinson's disease. Parkinsonism Relat Disord. 2014:20:637-9.

12. Cicero AF, Desideri G, Grossi G, Urso R, Rosticci M, D'Addato S, et al. Serum uric acid and impaired cognitive function in a cohort of healthy young elderly: data from the Brisighella study. Intern Emerg Med. 2015;10:25-31.

13. Mendez-Hernandez E, Salas-Pacheco J, Ruano-Calderon L, Tellez-Valencia A, Cisneros-Martinez J, Barraza-Salas M, et al. Lower uric acid linked with cognitive dysfunction in the elderly. CNS Neurol Disord Drug Targets. 2015;14:564-6.

14. Molshatzki N, Weinstein G, Streifler JY, Goldbourt U, Tanne D. Serum uric acid and subsequent cognitive performance in patients with pre-existing cardiovascular disease. PLoS One. 2015;10:e120862.

15. Tuven B, Soysal P, Unutmaz G, Kaya D, Isik AT. Uric acid may be protective against cognitive impairment in older adults, but only in those without cardiovascular risk factors. Exp Gerontol. 2017;89:15-9.

16. De Giorgi A, Fabbian F, Pala M, Tiseo R, Parisi C, Misurati E, et al. Uric acid: friend or foe? Uric acid and cognitive function "gout kills more wise men than simple". Eur Rev Med Pharmacol Sci. 2015;19:640-6.

17. Euser SM, Hofman A, Westendorp RG, Breteler MM. Serum uric acid and cognitive function and dementia. Brain. 2009;132:377-82.

18. Ruggiero C, Cherubini A, Lauretani F, Bandinelli S, Maggio M, Di lorio A, et al. Uric acid and dementia in community-dwelling older persons. Dement Geriatr Cogn Disord. 2009:27:382-9.

19. Li J, Dong BR, Lin P, Zhang J, Liu GJ. Association of cognitive function with serum uric acid level among Chinese nonagenarians and centenarians. Exp Gerontol. 2010;45:331-5.

20. Liu M, He Y, Jiang B, Wu L, Yang S, Wang Y, et al. Association between serum uric acid level and metabolic syndrome and its sex difference in a Chinese community elderly population. Int J Endocrinol. 2014:2014:754678.

21. $B L, T W, H n Z, W w Y, H p Y, C x L$, et al. The prevalence of hyperuricemia in China: a meta-analysis. BMC Public Health. 2011;11:832.

22. Xu G, Meyer JS, Huang Y, Du F, Chowdhury M, Quach M. Adapting minimental state examination for dementia screening among illiterate or minimally educated elderly Chinese. Int J Geriatr Psychiatry. 2003;18:609-16.

23. Peng D, Xu X, Liu J, Jiao Y, Zhuang H, Meng X, et al. Discussion on application of MMSE for senile dementia patients. Chinese Journal of Neuroimmunology and Neurology2005. 12:187-90.

24. Liu M, He Y, Jiang B, Wu L, Wang J, Yang S, et al. Association between metabolic syndrome and mild cognitive impairment and its age difference in a Chinese community elderly population. Clin Endocrinol. 2015;82:844-53.

25. Braga F, Pasqualetti S, Ferraro S, Panteghini M. Hyperuricemia as risk factor for coronary heart disease incidence and mortality in the general population: a systematic review and meta-analysis. Clin Chem Lab Med. 2016;54:7-15.

26. Galassi FM, Borghi C. A brief history of uric acid: from gout to cardiovascular risk factor. Eur J Intern Med. 2015:26:373.

27. Abeles AM. Hyperuricemia, gout, and cardiovascular disease: an update. Curr Rheumatol Rep. 2015:17:13. 